\title{
Individual decision making in task-oriented groups
}

\author{
Sandro M. Reia, ${ }^{1}$ Paulo F. Gomes, ${ }^{1,2}$ and José F. Fontanari ${ }^{1}$ \\ ${ }^{1}$ Instituto de Física de São Carlos, Universidade de São Paulo, \\ Caixa Postal 369, 13560-970 São Carlos, São Paulo, Brazil \\ ${ }^{2}$ Instituto de Ciências Exatas e Tecnológicas, Universidade Federal de Goiás, 75801-615 Jataí, Goiás, Brazil
}

\begin{abstract}
The strategies adopted by individuals to select relevant information to pass on are central to understanding problem solving by groups. Here we use agent-based simulations to revisit a cooperative problem-solving scenario where the task is to find the common card in decks distributed to the group members. The agents can display only a sample of their cards and we explore different strategies to select those samples based on the confidences assigned to the cards. An agent's confidence that a particular card is the correct one is given by the number of times it observed that card in the decks of the other agents. We use a Gibbs distribution to select the card samples with the temperature measuring the strength of a noise that prevents the agents to correctly rank the cards. The group is guaranteed to find the common card in all runs solely in the infinite temperature limit, where the cards are sampled regardless of their confidences. In this case, we obtain the scaling form of the time constant that characterizes the asymptotic exponential decay of the failure probability. For finite time, however, a finite temperature yields a probability of failure that is several orders of magnitude lower than in the infinite temperature limit. The available experimental results are consistent with the decision-making model for finite temperature only.
\end{abstract}

\section{INTRODUCTION}

A central goal of the study of psychologically-inspired problem-solving systems is the understanding and modeling of the human mental processes of decision making 1, 2. A second, not less important goal, is the evaluation and proposal of strategies to improve the quality of those decisions. Accordingly, in this paper we revisit and generalize a cooperative problem-solving scenario introduced by Leavitt in the early 1950s [3] (see also 4]), aiming at finding the decision-making strategies that optimize the performance of the group.

In Leavitt's problem-solving scenario, each of $N$ subjects is dealt a deck of $N$ cards selected from a master set of $N+1$ cards. The setup is such that each card appears on $N-1$ decks, except for one card that appears on all $N$ decks. The group's task is to find the common card in the shortest time possible 3. The original experiments employed $N=5$ subjects who were placed on cubicles that allowed the realization of a variety of communication patterns. Actually, this setup was originally devised and subsequently widely used to study the effects of the communication patterns on group performance [5] 8] (see [9, 10] for more recent contributions in this line). The subjects communicated by writing messages that could be passed through slots in the walls of the cubicles. The task was considered finished when each agent indicated the common card by throwing an appropriate switch.

The nature of Leavitt's task contrasts with the problems usually posed to cooperative problem-solving systems, which can also be solved by a single agent or by agents working independently of each other [11-15]. In fact, cooperation among the members of the group is mandatory to find the common card in the decks. In particular, the typical behavior of a subject in working toward a solution was to show all its cards to the members of the group with whom it was allowed to communicate, who then used that information to update their confidences about which card of their decks was the common card $[3$.

Rather than focusing on small groups of human subjects as in the seminal works of the $1950 \mathrm{~s}$, here we consider extensive agent-based simulations aiming at offering a complete perspective on the group performance for general $N$. In time, we assume that the group's choice of the common card is determined by the majority rule with eventual ties broken randomly, and that the group performance is measured by the failure probability, i.e., the probability the group chooses a wrong card.

In addition, we make Leavitt's task more difficult, as well as more interesting from the perspective of decision making, by limiting to $C \leq N$ the number of cards the agents (i.e., our virtual subjects) can display and by asking the group to make a decision at any time $\tau$. The important feature is that now the agents must also decide which cards to show to the other members of the group. Here we consider two possibilities: the agents simply sample $C$ cards at random from their decks or they pick the sample they estimate is the most likely to contain the correct card. Of course, an agent's confidence that a particular card is the correct one is determined by the number of times it observed that card in the decks of the other agents. These two possibilities can be viewed as extremes of a Gibbs probability distribution where the temperature $T$, which must be interpreted simply as a noise parameter, measures the strength of a random perturbation that prevents the agents to rank correctly the cards according to their confidences.

Since our interest here is on the evaluation of decisionmaking strategies rather than on the effects of communication patterns, we assume that an agent can, in principle, interact with any other member of the group, so that we select the pairs of interacting agents at random among the members of the group. Somewhat surpris- 
ingly, we find that the zero-temperature, noiseless limit, in which the agents display only their highest confidence cards, yields an extremely poor performance for any time $\tau$. In addition, we find that in the asymptotic time limit only the infinite temperature limit, in which the cards are displayed without regard to their confidences, guarantees that the correct card is found in all experiments. In this case, we find that the probability of failure decreases exponentially with increasing time and that the time constant $\tau_{c}$ scales with $N^{3 / 2}$ for $C<N$ and with $N$ for $C=N$. For a fixed time $\tau$, however, intermediate values of the temperature yield a performance several orders of magnitude superior to the performances of the two temperature extremes. This surprising result reveals the importance of the agent's decision-making strategies to the optimization of group performance.

The rest of this paper is organized as follows. In Section III we describe our generalization of Leavitt's task and the rules that govern the behavior of the agents in their attempts to complete the task. In Section III we present and analyze the results of the agent-based simulations, emphasizing the two extreme cases where the displayed cards are drawn randomly without regard to their confidences $(T \rightarrow \infty)$ and where they are chosen deterministically according to their confidence ranks $(T=0)$. Finally, in Section IV we summarize our main findings and compare them with the experimental results.

\section{MODEL}

We consider $N$ card decks where each deck contains $N$ distinct cards. The cards of a deck are selected from a collection of $N+1$ distinct cards, so that there is only one card that is common to all decks and any two decks have exactly $N-1$ cards in common. These decks are distributed to a group of $N$ agents (one deck for each agent) and the task of the group is to find the unique card that appears in all decks. The agents must achieve that by exchanging information about their card decks and formulating a strategy to decide each of their $N$ cards is the common one. Next we describe the interaction and decision-making processes.

Each agent $i=1, \ldots, N$ assigns a confidence value to each card $\alpha_{i}=1, \ldots, N$ in its deck, denoted by the integer variable $F_{i \alpha_{i}}^{\tau}=0,1, \ldots, \tau$, where $\tau$ stands for the number of interaction rounds or, more simply, the time. This quantity measures the confidence of agent $i$ that card $\alpha_{i}$ is the common card in the decks. The card labels have a subscript signaling the deck (or the agent) they belong to, since the decks do not have the same cards and there is no point for an agent to assign a confidence value to a card that is not in its deck as that card surely is not the card common to all decks. Hence, when agent $i$ is prompted to answer which card is the common one at time $\tau$, it selects the card that maximizes the confidences $F_{i \alpha_{i}}^{\tau}$. In case of a draw, a card is selected at random among the tied cards. Initially all cards have the same confidence values, viz., $F_{i \alpha_{i}}^{0}=0$ for all $i$ and $\alpha_{i}$. The effect of the interaction between agents is then to change these confidences, as we describe next.

We impose no restrictions on the communication between agents so, in principle, an agent can interact with all its $N-1$ peers in the group. More pointedly, at time $\tau$ we choose randomly two distinct agents in the group. The first agent - the observer - assesses $C$ cards of the second agent - the observed - and updates its confidences by adding one unit of confidence to the cards they have in common. An interaction round is complete when this procedure is repeated $N$ times, so that $N$, not necessarily distinct, agents have updated their confidences. (We note that only the observers update their confidences.) Then the time counter is incremented by one unit, i.e, $\tau \rightarrow \tau+1$.

Since it is the observed agent that selects the $C$ cards that are assessed by the observer, we need to set up strategies for selecting those cards. The most natural strategy is simply to sample $C$ cards without replacement from the deck of the observed agent. Another natural strategy is to choose the $C$ cards with the highest confidences. As already pointed out, these two strategies can be seen as extremes of the temperature of a Gibbs distribution as follows. We recall that there are $\left(\begin{array}{l}N \\ C\end{array}\right)$ different samples of $C$ cards that can be drawn from a deck of $N$ cards. Because each agent holds a different deck, we denote those samples by $\mathcal{C}_{k_{j}}$ with $k_{j}=1, \ldots,\left(\begin{array}{l}N \\ C\end{array}\right)$, where $j$ is the label of the agent. Then we associate an energy value to each sample,

$$
E\left(\mathcal{C}_{k_{j}}\right)=-\sum_{\alpha_{j} \in \mathcal{C}_{k_{j}}} F_{j \alpha_{j}}^{\tau},
$$

which is minimized by the sample containing the cards with the highest confidence values. Here we assume that a sample $\mathcal{C}_{k_{j}}$ is selected with probability

$$
\mathcal{P}\left(\mathcal{C}_{k_{j}}\right)=\frac{1}{Z_{j}} \exp \left[-\beta E\left(\mathcal{C}_{k_{j}}\right)\right]
$$

where $Z_{j}=\sum_{\mathcal{C}_{k_{j}}} \exp \left[-\beta E\left(\mathcal{C}_{k_{j}}\right)\right]$ is a normalization factor. The temperature $T=1 / \beta \geq 0$ is interpreted as a measure of a noise that prevents the observed agent to select accurately the cards with the highest confidences. This parameter allows us to interpolate between the random sampling of cards $(T \rightarrow \infty)$ and the selection of the cards with the highest confidences $(T=0)$.

Our introduction of the sample size $C=1,2, \ldots, N$ is justified when $N$ is large and so it would take too long for the observer to scan and record all cards in the hand of the observed agent. In any event, here we view it as an additional rule of the interaction between agents, i.e., that the observer can view only $C$ cards of the deck of the observed agent.

The decision of the group at time $\tau$ is obtained by asking each agent $i$ to designate its guess of the common card. As pointed out before, this is done by picking 
the card $\alpha_{i}$ that maximizes the confidences $F_{i \alpha_{i}}^{\tau}$. The group guess is then determined by the majority rule, i.e., the group picks the card designated by the majority of the agents. As usual, in case of a draw, the decision is made by selecting randomly one of the tied cards. Here we measure the group performance by the failure probability $P_{\tau}$, which is given by the fraction of independent experiments that failed to find the common card at time $\tau$. We use the failure probability, rather than the success probability $1-P_{\tau}$, because it is more convenient for the purpose of quantifying its time asymptotic behavior in the case of efficient decision strategies.

In sum, the group dynamics proceeds as follows. At $\tau=0$ we assign the card decks to the agents and each agent sets to zero the confidences of its cards. Next we choose randomly the observer and the observed agent. The observed agent evaluates the energy of each of the $\left(\begin{array}{l}N \\ C\end{array}\right)$ samples of $C$ cards and picks a sample with a probability given by the Gibbs distribution (2). The observer then updates the confidences of the cards that appear in the sample selected by the observed agent (i.e., the cards common to the sample and to the observer's deck). The time $\tau$ increases by one unit only after this procedure is repeated $N$ times.

\section{RESULTS}

The initial setup of the $N$ card decks is deterministic, i.e., all experiments begin with the same decks, as described in Section III. The experiments have two sources of stochasticity, viz., the choice of the pairs of interacting agents and the sample of $C$ cards scanned by the observer. In the case $C=N$, stochasticity is due only to the selection of the interacting agents. The results presented here represent averages over $10^{5}$ independent runs. Next we discuss separately from the general case the two temperature extremes of the Gibbs distribution (2) as their study does not require the time-consuming generation of the $\left(\begin{array}{l}N \\ C\end{array}\right)$ cards samples.

\section{A. Infinite temperature limit}

Here we consider the case where the observed agent randomly chooses a sample of $C$ cards from its deck without regard to their confidences, and offers them to the observer for inspection. This corresponds to the limit $T=1 / \beta \rightarrow \infty$ of the Gibbs distribution (2). This information is then used to update the observer's cards confidences as discussed before. Figure 1 shows the dependence of the probability of failure $P_{\tau}$ on the time $\tau$ for fixed $N$ in a semi-log plot. Except for small $\tau$, this probability exhibits a neat exponential decay characterized by the time constant $\tau_{c}=\tau_{c}(N, C)$, which measures the typical time the group requires to complete successfully the task. This figure shows that, as expected, $\tau_{c}$ increases as the sample size $C$ decreases and, more im-

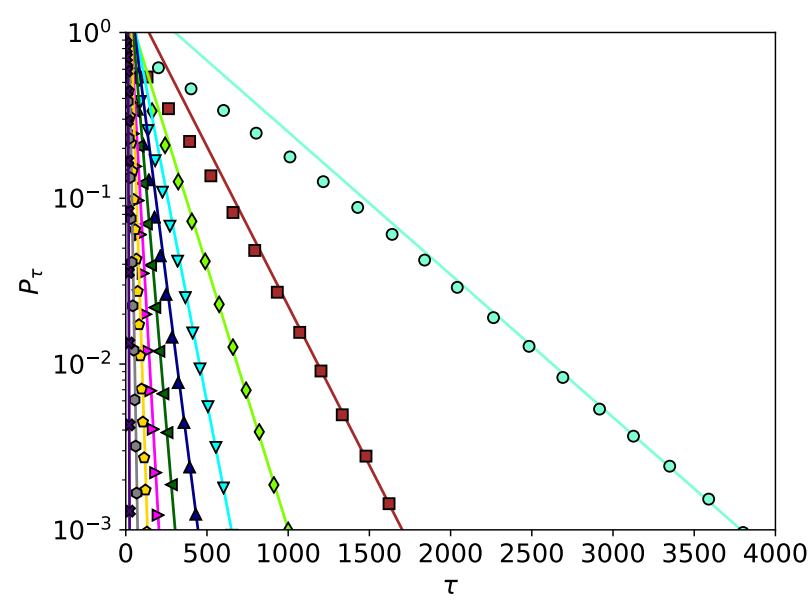

FIG. 1. Failure probability $P_{\tau}$ at time $\tau$, for $N=10$ and (right to left) $C=1,2, \ldots, 10$ in the infinite temperature limit. The lines are the fittings $P_{\tau}=a_{N, C} \exp \left(-\tau / \tau_{c}\right)$ where $a_{N, C}$ and $\tau_{c}=\tau_{c}(N, C)$ are fitting parameters.

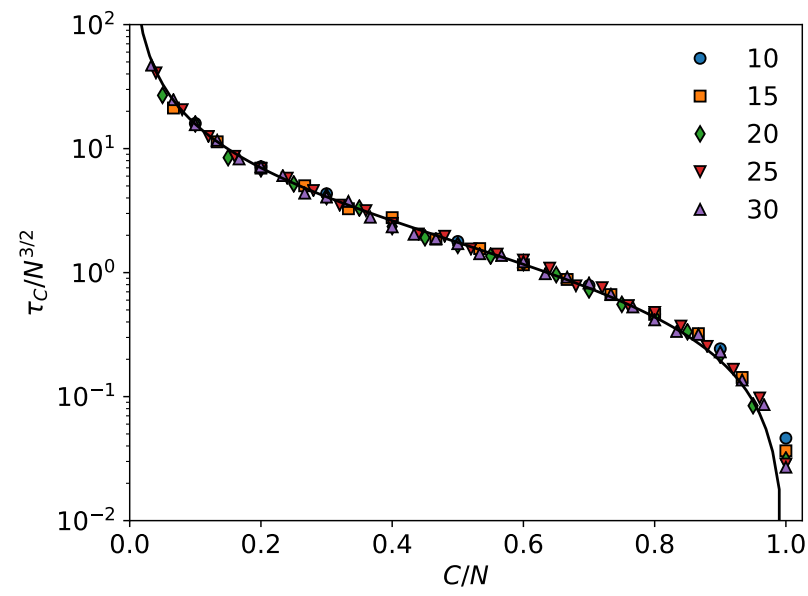

FIG. 2. Semi-logarithm plot of the time constant $\tau_{c}$ as function of the ratio $C / N$ for the infinite temperature limit and $N=10,15,20,25,30$ as indicated. The curve is the fitting $\tau_{c}=1.75[1 /(C / N)-1]$.

portantly, that given enough time the group is always guaranteed to find the common card, i.e., $P_{\tau} \rightarrow 0$ for $\tau \rightarrow \infty$

The dependence of the time constant $\tau_{c}$ on the parameters of the model is exhibited more clearly in Fig. 2, which shows that the data for different $N$ are described very well by the scaling form

$$
\tau_{c}=N^{3 / 2} f(C / N),
$$

with the scaling function $f(x)=1.75(1 / x-1)$. In this figure we use a semi-logarithm plot to highlight the goodness of fit in the region of $C / N$ close to 1 . We note that in the case $C=N, \tau_{c}$ increases linearly with increasing $N$ as shown in Fig. 3. This result is consistent with 


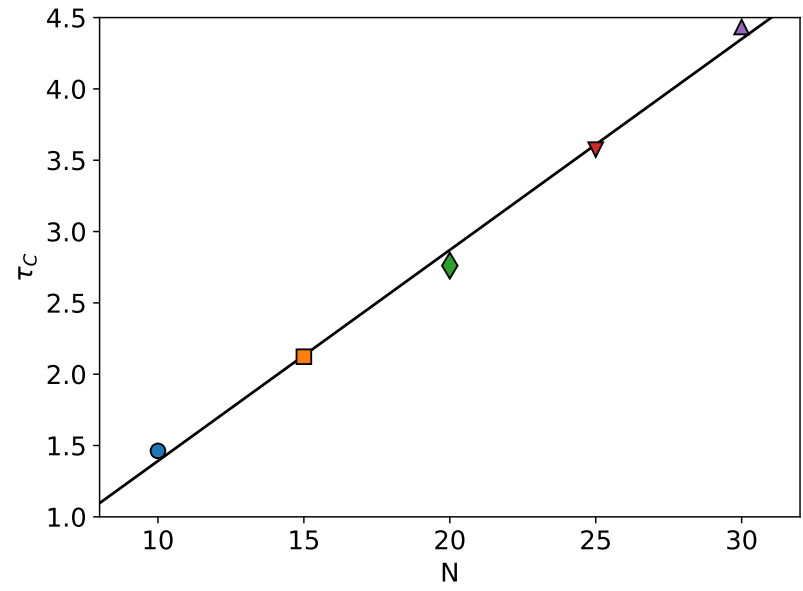

FIG. 3. Dependence of the time constant $\tau_{c}$ on the group or deck size $N$ for $C=N$ in the infinite temperature limit. The symbols convention is the same as for Fig. 2. The straight line is the fitting $\tau_{c}=-0.09+0.15 \mathrm{~N}$.

eq. (3) because $f(1)=0$ and so this equation does not determine the scaling of $\tau_{c}$ with $N$ in the case $C=N$. Interestingly, the uncertainty introduced by reducing the amount of information the agents can transmit to each other changes the scale of the time constant with the group or deck size.

\section{B. Zero temperature limit}

Since in each interaction the observer inspects only $C$ cards of the deck of the observed agent, a natural strategy to improve the performance of the group is to guarantee that the latter displays the sample of cards that is the most likely to contain the correct card. An apparently sensible strategy is to choose the $C$ cards with the highest confidence values. This can be achieved by considering the zero temperature $T=1 / \beta \rightarrow 0$ limit of the Gibbs distribution (2), but it is much more efficient simply to rank the card confidences $F_{j \alpha_{j}}^{\tau} ; \alpha_{j}=1, \ldots, N$ of the observed agent $j$ and select the top $C$ cards. Eventual draws between cards in and out this selection are broken randomly, as usual.

We find that displaying the $C$ cards with the highest confidences is actually disastrous since the probability of failure rapidly tends to a nonzero limiting value and becomes insensitive to increasing $\tau$. (This point will become clearer in the study of the finite temperature case.) Accordingly, in Fig. 4 we show the asymptotic failure probability $P_{\infty}$ for several deck sizes $N$. Extrapolation of these data to $N \rightarrow \infty$ yields the simple result $P_{\infty}=1-C / N$, which offers a clue to the failure of this noiseless strategy. In fact, there is the sporting chance $(N-C) / N=1-C / N$ that the common card is not chosen by an agent in the first round when the confidences

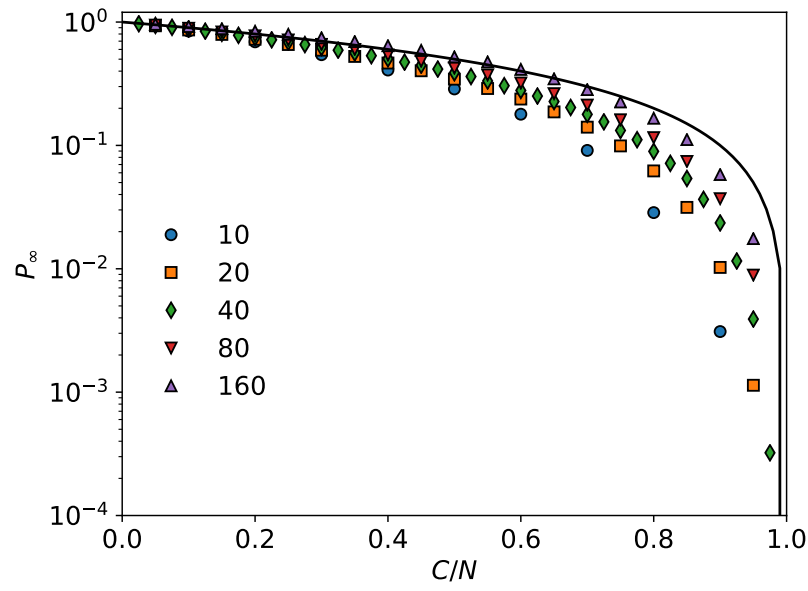

FIG. 4. Asymptotic probability of failure $P_{\infty}$ as function of the ratio $C / N$ for the zero temperature limit and $N=$ $10,20,40,80,160$ as indicated. The solid curve is the function $P_{\infty}=1-C / N$ obtained by extrapolating the finite $N$ results to $N \rightarrow \infty$.

are equiprobable and the results indicate that for large $N$ this agent can strongly influence the other agents towards a wrong decision.

\section{Finite temperature}

Here we consider the more general scheme that encompasses both strategies presented before, in which the $C$ cards shown to the observer are selected according to the Gibbs distribution (2) using a finite and nonzero temperature. The implementation of this case is more demanding computationally as we need to calculate the probabilities associated to all $\left(\begin{array}{l}N \\ C\end{array}\right)$ samples of $C$ cards that can be drawn from the deck of the observed agent.

Figure 5, which shows the probability of failure $P_{\tau}$ as function of the inverse temperature $\beta$ at different times $\tau$, reveals a few surprises. First, except for very short times the noiseless strategy exhibits the worst performance, as mentioned before. Second, for fixed $\tau$ there is a nonzero value of $\beta$ that minimizes the failure probability. For instance, in Fig. 5 the failure probability for $\tau=363$ at $\beta=0.01$ is several orders of magnitude lower than at $\beta=$ 0 . Third and most surprisingly, the asymptotic failure probability vanishes for $\beta=0$ only. More pointedly, we find $P_{\infty} \asymp \exp (-b / \beta)$ where $b$ is a parameter that depends on $N$ and $C$. This means that the value of $\beta$ that minimizes $P_{\tau}$ tends to zero as $\tau$ increases, a trend that is already visible in the figure. We have verified that these conclusions hold true for all values of the parameters $N$ and $C<N$. 


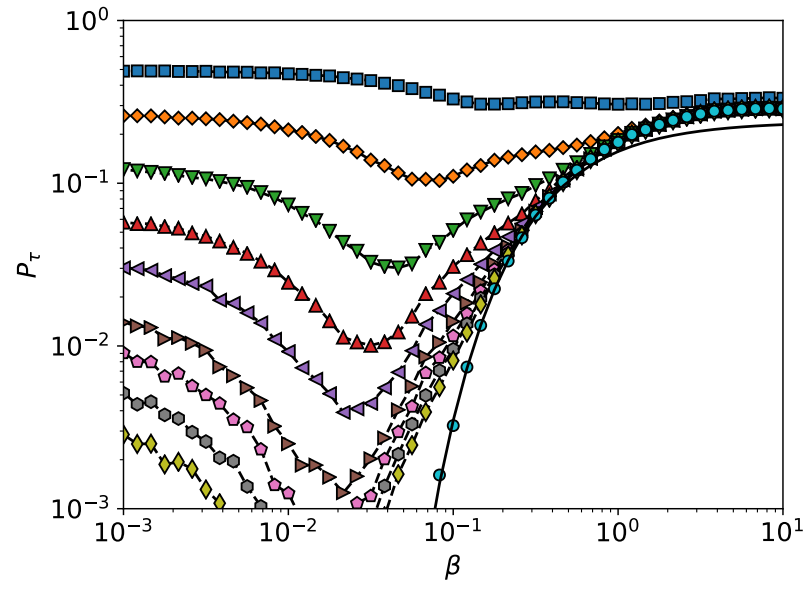

FIG. 5. Probability of failure $P_{\tau}$ as function of the inverse temperature $\beta$ for $N=10$ and $C=5$ for (top to bottom) $\tau=$ $43,91,144,190,229,275,301,331,363$ and $10^{4}$. In the limit $\beta \rightarrow \infty$, we find $P_{\infty}=0.287$. The solid curve is the fitting of the data for $\tau=10^{4}, P_{10^{4}}=a \exp (-b / \beta)$ where the fitting parameters are $a=0.251$ and $b=0.428$.

\section{DISCUSSION}

Understanding the influences on group performance is a central issue in collective intelligence 16. Most theoretical and experimental efforts have focused on the influence of the composition of the group, since this factor can be more easily observed and controlled [17, 18. Here we focus instead on the strategies adopted by individuals to select and transmit relevant information to the other members of the group.

More pointedly, we revisit Leavitt's cooperative problem-solving scenario in which each member of a group of size $N$ receives a deck with $N$ cards and its task is to find the card that is common to all decks in the shortest time [3, 4]. The decks are contrived so as to maximize the difficulty of the task, e.g., any two decks have $N-1$ cards in common and the cards come in $N+1$ types. Whereas the experiments carried out in the 1950 s employed $N=5$ subjects in a variety of communication patterns (i.e., who can communicate with whom), here we use agent-based simulations to study the group performance for general $N$ in a fully connected communication net. We ground our study on the persuasive assumption that an agent's confidence that a particular card is the correct one is determined by the number of times it observed that card in the decks of the other agents. In fact, this strategy of increasing the card confidences following the agent interactions is akin to the scheme used to model lexicon acquisition in a cross-situational learning scenario [19, 20. In addition, we assume that the group decision is determined by applying the majority rule to the decisions of the group members.

In the case the agents can assess all cards of the decks of the other members, we find that the scale of time to complete successfully the task grows linearly with the number of agents or decks, i.e., $\tau_{c} \propto N$ (see Fig. 3). More pointedly, we find that the probability of failure decreases exponentially with increasing time, $P_{\tau} \propto \exp \left(-\tau / \tau_{c}\right)$. This exponential decay is a consequence of the fact that the interacting agents are chosen randomly, leading to the possibility of uninformative repeated interactions or of one or more agents never being selected for interaction in a finite time $\tau$.

The group task is made considerably more difficult by limiting to $C<N$ the number of cards that the agents can show to each other. In fact, in the case the $C$ cards are sampled randomly without regard to their confidences, the time constant becomes much greater than in the unrestricted case $C=N$, namely, $\tau_{c} \propto N^{3 / 2}$ (see Fig. 2). An interesting consequence of restricting the number of cards displayed is the need to specify a strategy to select those cards. A natural line that we pursue here is to link the chances of display of a card to its confidence. We find that the exhibition of the cards with the highest confidences yields a very poor performance since it maximizes the failure probability for large $\tau$ (see Fig. 5). The importance of the confidences on the selection of the displayed cards can be tuned by introducing the Gibbs probability distribution (2), where the temperature $T=1 / \beta$ measures the strength of a noise that prevents the correct ranking of the cards. Most interestingly, for fixed $\tau$ we find that the failure probability is minimized for a finite, nonzero temperature (see Fig. 5). However, in the asymptotic limit $\tau \rightarrow \infty$ it vanishes only in the infinite temperature limit, i.e., in the case the cards confidences do not affect their chances of being selected for display.

Although the two temperature extremes of the Gibbs distribution (2) are sensible strategies the subjects could adopt in a real-world situation, the finite temperature strategies are clearly too complex to be used in practice. Of course, our intention of introducing the noise parameter or temperature was simply to add an element that could prevent the correct ranking of the cards and, as physicists, the Gibbs distribution came along naturally. We expect, however, that a more psychologically realistic implementation of this noise would produce similar results.

Despite the simplicity of Leavitt's task, it does not yield to an analytical approach even in the infinite temperature limit, where the card confidences play no role on the decisions of the agents. In fact, a natural procedure to tackle this problem analytically is to write recursion equations for the probabilities that the card confidences take on the (integer) values $0, \ldots, \tau$ at time $\tau$ [21]. The difficulty is that the card confidences are not independent random variables and so we need to work with the joint probability distribution of the $N$ card confidences, which is impractical even for small $N$. Hence our Monte Carlo approach is probably the simplest, if not the only, way to study Leavitt's task and its generalizations proposed 
TABLE I. Asymptotic mean number of runs with at least one error in the case of $N=5$ agents fixed at the corners of a pentagon. The experimental result is $\eta=0.23[3$.

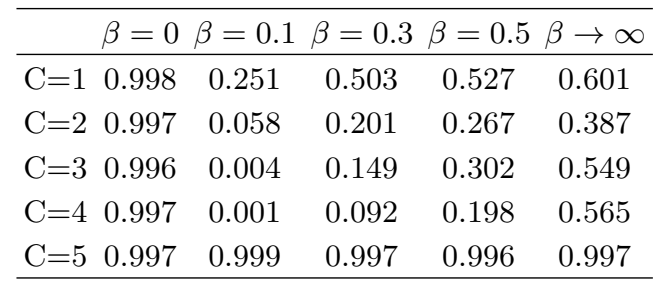

here. Nevertheless, our use of scaling forms to analyze the simulation results, which is perhaps the main contribution of physics to the so-called science of complexity 22], offered a thorough characterization of the problem.

To conclude, a comparison of our results with the seminal experiments of Leavitt [3] is in order. As pointed out, the experimental studies considered $N=5$ subjects, who were positioned at the corners of geometric patterns (e.g., a pentagon). In addition, there were no restrictions on the number of cards that could be displayed (i.e., $C$ was not fixed and could vary in time as well as from subject to subject). More importantly, Leavitt did not focus on the group decision as we did here, but on the individual subjects' decisions. An unambiguous outcome of Leavitt's experiments is the mean number of experiments with at least one error, which we denote by $\eta$. An error occurs when a subject selects a wrong card. In the case of a pentagon, Leavitt found $\eta=0.23$. Our simulation results for this scenario are exhibited in Table I where we varied the sample size $C$ and the inverse temperature $\beta$. The results indicate that for any value of $C<5$ it is possible to find a value of $\beta$ that reproduces the experimental result, which, however, cannot be reproduced by any of the temperature extremes. This finding highlights the importance of (moderate) noise to model the human mental processes of decision making.

The simplicity of Leavitt's task and the prospect of modeling the decision-making process of the individuals is an enthralling research avenue that calls for more experimental results using, perhaps, digital platforms that are not limited by the physical constraints of the experiments carried out in the 1950s. We hope this contribution will motivate further research on this direction.

\section{ACKNOWLEDGMENTS}

The research of JFF was supported in part by Grant No. 2017/23288-0, Fundação de Amparo à Pesquisa do Estado de São Paulo (FAPESP) and by Grant No. 305058/2017-7, Conselho Nacional de Desenvolvimento Científico e Tecnológico (CNPq). SMR was supported by grant 15/17277-0, Fundação de Amparo à Pesquisa do Estado de São Paulo (FAPESP).
[1] H.A. Simon, Models of Man: Social and Rational (John Wiley \& Sons, New York, 1957)

[2] A. Newell, Unified Theories of Cognition (Harvard University Press, Cambridge, MA, 1994)

[3] H.J. Leavitt, J. Abnorm. Soc. Psych. 46, 38 (1951)

[4] A. Bavelas, J. Acoustical Soc. Amer. 22, 725 (1950)

[5] G.A. Heise, G.A. Miller, J. Abnorm. Soc. Psych. 46, 327 (1951)

[6] H. Guetzkow, H.A. Simon, Manage. Sci. 1, 233 (1955)

[7] M.E. Shaw, J. Abnorm. Soc. Psych. 49, 547 (1954)

[8] M. Mulder, Sociometry 23, 1 (1960)

[9] W. Mason, D.J. Watts, Proc. Natl. Acad. Sci. 109, 764 (2012)

[10] S.M. Reia, S. Herrmann, J.F. Fontanari, Phys. Rev. E 95, 022305 (2017)

[11] S.H. Clearwater, B.A. Huberman, T. Hogg, Science 254, 1181 (1991)

[12] J. Kennedy, Adapt. Behav. 7, 269 (1999)

[13] D. Lazer, A. Friedman, Admin. Sci. Quart. 52, 667 (2007)
[14] J.F. Fontanari, Eur. Phys. J. B 88, 251 (2015)

[15] J.F. Fontanari, Cogn. Syst. Res. 50, 29 (2018)

[16] S.E. Page, The Difference: How the Power of Diversity Creates Better Groups, Firms, Schools, and Societies (Princeton University Press, Princeton, 2007)

[17] J.M. Levine, L.B. Resnick, E.T. Higgins, Annu. Rev. Psychol. 44, 585 (1993)

[18] A.W. Woolley, C.F. Chabris, A. Pentland, N.H.T. Malone, Science 333, 686 (2010)

[19] P.F.C. Tilles, J.F. Fontanari, EPL 99, 60001 (2012)

[20] R. Reisenauer, K. Smith, R.A. Blythe, Phys. Rev. Lett. 110, $258701(2013)$

[21] W. Feller, An Introduction to Probability Theory and Its Applications Vol. 1 (Wiley, New York, Third Edition, 1968)

[22] G.B. West, Scale: The Universal Laws of Growth, Innovation, Sustainability, and the Pace of Life in Organisms, Cities, Economies, and Companies (Penguim Press, New York, 2017) 